\title{
Relaxation Strategy for the Landweber Method
}

\author{
Guanghui $\operatorname{Han}^{\mathrm{a}}$, Gangrong $\mathrm{Qu}^{\mathrm{a}, \mathrm{b}, *}$, Ming Jiang ${ }^{\mathrm{c}, \mathrm{d}, *}$ \\ ${ }^{a}$ School of Science, Beijing Jiaotong University, Beijing 100044, China \\ ${ }^{b}$ Beijing Center for Mathematics and Information Interdisciplinary Sciences (BCMIIS), Beijing 100048, China \\ ${ }^{c}$ LMAM, School of Mathematical Sciences, Peking University, Beijing 100871, China \\ ${ }^{d}$ Cooperative Medianet Innovation Center, Peking University, Beijing 100871, China
}

\begin{abstract}
The Landweber iteration is a general method for the solution of linear systems which is widely applied for image reconstructions. The convergence behavior of the Landweber iteration is of both theoretical and practical importance. By the representation of the iterative formula and the convergence results of the Landweber iteration, we derive the optimal relaxation method under the minimization of the spectral radius of the newly derived iterative matrix. We also establish the iterative relaxation strategy to accelerate the convergence for the Landweber iteration when only the biggest singular value is available. As an immediate result, we derive the corresponding results for the Richardson's iteration for the symmetric nonnegative definite linear systems. Finally, numerical simulations are conducted to validate the theoretical results. The advantage of the proposed relaxation strategies is demonstrated by comparing with the existing strategies.

Keywords: Landweber iteration, relaxation strategy, image reconstruction, Weighted least-squares.
\end{abstract}

\section{Introduction}

Many application problems, for example, image reconstruction can be modeled as the following linear systems

$$
A x=b
$$

where $b=\left(b^{1} \cdots b^{M}\right)^{\mathrm{T}} \in \mathbf{K}^{M}$ is the observed data with the noise or noise-free, $x=\left(x_{1} \cdots x_{N}\right)^{\mathrm{T}} \in$ $\mathbf{K}^{N}$ is the solution, ${ }^{T}$ denotes the transpose of a vector or matrix. Here the number field $\mathbf{K}$ can be the reals $\mathbf{R}$ or the complexes $\mathbf{C}$ and the system matrix $A=\left(A_{i, j}\right)$ is nonzero with the dimension $M \times$ $N$. For image reconstruction problems, simultaneous iterative algebraic reconstruction methods are deduced to solve (1). The extensively used scheme is the following weighted Landweber scheme $[1-4,6-11]$

$$
x^{(n+1)}=x^{(n)}+\lambda_{n} V^{-1} A^{*} W\left(b-A x^{(n)}\right),
$$

\footnotetext{
* Corresponding author

Email addresses: grqu@bjtu.edu.cn (Gangrong Qu), ming-jiang@ieee.org (Ming Jiang)
} 
for $n=0,1, \cdots$, where $\lambda_{n}$ is called the relaxation coefficient, $x^{(0)}$ is an initial guess, $A^{*}$ is the conjugate transpose of $A, V$ and $W$ are two positive definite matrices with orders $N$ and $M$. For simplicity, we study the Landweber scheme without weights

$$
x^{(n+1)}=x^{(n)}+\lambda_{n} A^{*}\left(b-A x^{(n)}\right),
$$

because if we let $y^{(n)}=V^{\frac{1}{2}} x^{(n)}, D=W^{\frac{1}{2}} A V^{-\frac{1}{2}}, \bar{b}=W^{\frac{1}{2}} b$, then the weighted Landweber scheme (2) can be transformed to the Landweber scheme without weights [3,21]. Generally, the linear systems (1) is inconsistent. The Landweber iteration is to find the least-square solutions of (1). The least-squares functional

$$
L(x)=\frac{1}{2}\|b-A x\|^{2}
$$

is minimized to find a solution. The gradient of $L$ is

$$
\nabla L(x)=-A^{*}(b-A x)
$$

All the minimizers of $L$ satisfy the following normal equation

$$
A^{*} A x=A^{*} b,
$$

and each solution of (6) is a minimizer of the least-squares function $L$. The equation (6) is always solvable and has a minimal 2-norm solution $x^{+}$, which is equal to $A^{+} b$, where $A^{+}$is the MoorePenrose inverse of $A$ [15]. The Landweber scheme (2) is a preconditioned gradient based method for the least-squares functional. It is proved that the Landweber scheme converges to the solution $x^{+}+P^{A}\left(x^{(0)}\right)$ of the normal equation (6) under convergence conditions [2,3,4,25], where $P^{A}\left(x^{(0)}\right)$ is the projection of initial guess $x^{(0)}$ on the null space of $A$.

The principal questions about any iterative method are its convergence behavior and computational stability, which often affect the quality of the reconstructed image. In $[2,3,4,12]$, the convergence conditions of the Landweber scheme in both simultaneous and block-iterative cases were established for variant relaxation coefficients. As demonstrated [14], the convergence rate of the Landweber scheme was shown to be linear if the relaxation coefficients are constant. The necessary and sufficient conditions for the convergence of the iteration $x^{(n)}$ and for its convergence to a solution of the normal equation (6) are presented in [7]. A neat iterative representation formula was derived in [21] and the convergence conditions in [7] were refined in [21].

The motivations of this work are to find the optimal relaxation coefficient if the biggest and the smallest positive eigenvalues are known, and to find a relaxation strategy to accelerate the convergence if only the biggest eigenvalue is available. The following is a review of the advances on the simultaneous iterative algebraic reconstruction methods.

In $[18,19,25]$, when the matrix $A^{*} A$ is positive definite, the optimal relaxation method of the Landweber iteration, as a special case of the Richardson iteration, is given by $\lambda_{n}=\frac{2}{\mu_{\max }+\mu_{\min }}(n=$ 
$1,2, \cdots)$, where $\mu_{\max }$ and $\mu_{\min }$ are the biggest and the smallest eigenvalues of $A^{*} A$. However, when the matrix $A^{*} A$ is only semi-positive definite and singular, we can prove that the iteration $x^{(n)}$ generated by the Landweber scheme $(3)$ with $\lambda_{n}=\frac{2}{\mu_{\max }+\mu_{\min }}(n=1,2, \cdots)$ is divergent and does not converge to the solution $x^{+}+P^{A}\left(x^{(0)}\right)$. Please refer to Theorem 2.2 (c) for details.

In [27], an eigenvalue based scheme is established to automatically determine the near-optimal value of the relaxation parameter to accelerate the convergence rate of SIRT so that only half the number of iterations normally required is needed. In [28], the optimal parameters are chosen to make the errors in projected data as well as image errors are minimal. A new "training" algorithm that finds the optimal parameter for a given test problem and a MATLAB package with implementations of SIRT were provided in [29]. In [30,31], the relaxation parameter strategies of controlling the propagated noise component of the error and the semi-convergence for Simultaneous Iterative Reconstruction Technique (SIRT) and the projected Landweber algorithm were proposed. The strategies are competitive when the noise is not too small. Another strategy is based on picking $\lambda_{n}$ such that the error $\left\|x^{*}-x^{(n)}\right\|$ is minimized in each iteration, where $x^{*}$ is a solution to $A x=b$ [32]. Similar strategies have also been proposed in [33,34]. Recently, the optimal relaxation problem was revisted. Two near-optimal relaxations based on eigenvalue bounds and a heuristic extension for ordered sub-sets was proposed. It was demonstrated that the SIRT and the separable quadratic surrogates (SQS) converge at the same rate [35]. For the image reconstruction with under-sampled data, an effective algorithm to accomplish multi-view learning with incomplete views by assuming that different views are generated from a shared subspace is reported in [36].

In this paper, with the neat iterative representation formula for the Landweber iteration in [21], we derive a new iterative representation (13) and find the optimal relaxation as $\lambda_{n}=\frac{2}{\mu_{1}+\mu_{m}}$, by minimizing the spectral radius of the newly derived iterative matrix at each iteration, where $\mu_{1}$ and $\mu_{m}$ are the biggest and the smallest positive eigenvalues of matrix $A^{*} A$. According to the optimal relaxation strategy and the convergence conditions, if only $\mu_{1}$ is known, we prove that the spectral radius of the derived iterative matrix with a relaxation coefficient $\lambda^{(1)} \in\left(0, \frac{1}{\mu_{1}}\right)$ is bigger than that of the relaxation coefficient $\lambda^{(2)}=\frac{2}{\mu_{1}}-\lambda^{(1)} \in\left(\frac{1}{\mu_{1}}, \frac{2}{\mu_{1}}\right)$. Hence, we propose the accelerating convergence relaxation strategies by $\lambda_{n} \in\left(\frac{1}{\mu_{1}}, \frac{2}{\mu_{1}}\right)$ and prove that the $\lambda_{n}$ satisfies the convergence conditions. Moreover, as an immediate result of the Landweber iteration, we obtain a generalization of the optimal relaxation for the Richardson iteration for symmetric nonnegative definite matrices.

This paper is organized as follows. In section 2, we introduce the notations and some preliminary results from linear algebra and describe the new representation of the Landweber iteration. Then the optimal and accelerating convergence scheme are proposed in section 3 . In section 4 , we 
derive the corresponding optimal relaxation strategy for the Richardson iteration of symmetric nonnegative definite linear systems. In section 5, we use the Cimmino's and DROP methods to simulate image reconstruction and compare with the existing relaxation strategies. Finally, we discuss some relevant issues and conclude this paper.

\section{Preliminaries}

In this section, we will use the notations and results derived in [21] and briefly state these here. We use * to denote the transpose of the matrix $A$ when $A$ is real, or the conjugate transpose of the matrix $A$ when $A$ is complex. We use $\mathbf{K}^{N}$ to denote the Hilbert space with the canonical inner product $\langle\cdot, \cdot\rangle$. The orthogonal complement of a subspace $E$ in $\mathbf{K}^{N}$ is denoted by $E^{\perp}$. The orthogonal projection from $\mathbf{K}^{N}$ to the null space $\mathcal{N}(A)$ is denoted by $P^{A}$. The zero element of the vector space $\mathbf{K}^{N}$ is denoted by $\theta$. By the orthogonal decomposition theorem $\mathbf{K}^{N}=\mathcal{N}(A)+\mathcal{N}(A)^{\perp}$. The same definitions and notations are applicable to $\mathbf{K}^{M}$.

Let $\left\{\mu_{k}\right\}_{k=1}^{m}$ be the distinct positive eigenvalues of $A^{*} A$ that are ordered such that $\mu_{1}>\cdots>$ $\mu_{m}>0$. Each $\mu_{k}$ has the algebraic multiplicity $p_{k}$, respectively, for $1 \leq k \leq m$. For $1 \leq j \leq p_{k}$, we let $u_{j, k}$ be the corresponding orthonormal eigenvectors. It follows by definition that

$$
A^{*} A u_{j, k}=\mu_{k} u_{j, k} .
$$

If 0 is an eigenvalue of $A^{*} A$ with an algebraic multiplicity $q$, let $v_{j}$ be the corresponding orthonormal eigenvectors for $1 \leq j \leq q$. Then $A^{*} A v_{j}=\theta$. With the above notations, the spectral norm or the 2-norm of the matrix $A$ is

$$
\|A\|^{2}=\mu_{1}
$$

Let $U_{k}=\left(u_{1, k}, \cdots, u_{p_{k}, k}\right)$ for $k=1, \cdots, m, V=\left(v_{1}, \cdots, v_{q}\right)$, and $U=\left(U_{1}, \cdots, U_{m}, V\right)$, then we have

$$
U^{*} A^{*} A U=\operatorname{Diag}\left(\mu_{1} I_{p_{1}}, \cdots, \mu_{m} I_{p_{m}}, O_{q}\right),
$$

where $I_{k}$ represents the $k$-order unit matrix and $O_{q}$ denotes the $q$-order zero matrix. The eigenvectors $\left\{u_{j, k}: 1 \leq j \leq p_{k}, 1 \leq k \leq m\right\} \cup\left\{v_{j}: 1 \leq j \leq q\right\}$ form an orthonormal basis of $\mathbf{K}^{N}$ and the vectors $\left\{u_{j, k}: 1 \leq j \leq p_{k}, 1 \leq k \leq m\right\}$ are an orthonormal basis of $\mathcal{N}(A)^{\perp}$; the vectors $\left\{v_{j}: 1 \leq j \leq q\right\}$ form an orthonormal basis of $\mathcal{N}(A)$. The minimal 2-norm solution $x^{+}$of $(6)$ is in the subspace $\mathcal{N}(A)^{\perp}$ and can be written as [15]

$$
x^{+}=\sum_{k=1}^{m} \sum_{j=1}^{p_{k}} c_{j, k} u_{j, k}
$$

with $c_{j, k} \in \mathbf{K}$, for $1 \leq k \leq m$ and $1 \leq j \leq p_{k}$. From (9) and (10), we get

$$
A^{*} b=A^{*} A x^{+}=\sum_{k=1}^{m} \mu_{k} \sum_{j=1}^{p_{k}} c_{j, k} u_{j, k} .
$$


The initial guess $x^{(0)}$ can be decomposed as

$$
x^{(0)}=\sum_{k=1}^{m} \sum_{j=1}^{p_{k}} c_{j, k}^{(0)} u_{j, k}+P^{A}\left(x^{(0)}\right)=x_{1}^{(0)}+P^{A}\left(x^{(0)}\right),
$$

with $c_{j, k}^{(0)} \in \mathbf{K}$, for $1 \leq k \leq m$ and $1 \leq j \leq p_{k}$. The representation of the iteration $x^{(n)}$ is characterized in the following theorem.

Theorem 2.1. 1 For $n=1, \cdots$, the $n$-th iteration $x^{(n)}$ generated by the Landweber scheme (3) satisfies

$$
\begin{aligned}
& x^{(n)}-x^{+}-P^{A}\left(x^{(0)}\right)=\prod_{s=0}^{n-1}\left(I-\lambda_{s} A^{*} A\right)\left(x_{1}^{(0)}-x^{+}\right) \\
= & U \operatorname{Diag}\left(\prod_{s=0}^{n-1}\left(1-\lambda_{s} \mu_{1}\right) I_{p_{1}}, \cdots, \prod_{s=0}^{n-1}\left(1-\lambda_{s} \mu_{m}\right) I_{p_{m}}, O_{q}\right) r,
\end{aligned}
$$

where $r=\left(r_{1,1}, \cdots, r_{1, p_{1}}, \cdots, r_{m, 1}, \cdots, r_{m, p_{m}}, 0, \cdots, 0\right)^{T} \in \mathbf{K}^{N}$ with $r_{j, k}=c_{j, k}-c_{j, k}^{(0)}$ for $1 \leq j \leq$ $p_{k}, 1 \leq k \leq m$ and $p=p_{1}+\cdots+p_{m}$.

Proof. From (11),

$$
x^{(n+1)}-x^{+}=x^{(n)}-x^{+}+\lambda_{n} A^{*} A\left(x^{+}-x^{(n)}\right)=\left(I-\lambda_{n} A^{*} A\right)\left(x^{(n)}-x^{+}\right) .
$$

So

$$
x^{(n)}-x^{+}=\prod_{s=0}^{n-1}\left(I-\lambda_{s} A^{*} A\right)\left(x^{(0)}-x^{+}\right) .
$$

By (11),

$$
x^{(n)}-x^{+}-P^{A}\left(x^{(0)}\right)=\prod_{s=0}^{n-1}\left(I-\lambda_{s} A^{*} A\right)\left(x_{1}^{(0)}-x^{+}\right),
$$

because of $A^{*} A P^{A}\left(x^{(0)}\right)=0$. From (11) and (12),

$$
x_{1}^{(0)}-x^{+}=\left(U_{1}, \cdots, U_{p}, V_{q}\right) r .
$$

By (9),

$$
x^{(n)}-x^{+}-P^{A}\left(x^{(0)}\right)=U \operatorname{Diag}\left(\prod_{s=0}^{n-1}\left(1-\lambda_{s} \mu_{1}\right) I_{p_{1}}, \cdots, \prod_{s=0}^{n-1}\left(1-\lambda_{s} \mu_{m}\right) I_{p_{m}}, I_{q}\right) U^{*}\left(U_{1}, \cdots, U_{p}, V_{q}\right) r .
$$

Therefore, theorem holds immediately.

We will use the following convergence results proposed in [21] and rewrite them in Theorem 2.2. Let $\|A\|_{2}=\rho>0$ and

$$
a_{1, n}=\min \left(\lambda_{n} \rho^{2}, 2-\lambda_{n} \rho^{2}\right),
$$

where $\mu_{1}=\rho^{2}$. By Theorem 2.1 and the orthonormal properties of the eigenvectors, we have the following theorem.

\footnotetext{
${ }^{1}$ Theorem 2.1 is the modification for the Theorem III.1 in [21].
} 
Theorem 2.2. (a) For $n=1, \cdots$,

$$
\begin{gathered}
x^{(n)}-x^{+}-P^{A}\left(x^{(0)}\right)=-\sum_{k=1}^{m} \prod_{s=0}^{n-1}\left(1-\lambda_{s} \mu_{k}\right) \sum_{j=1}^{p_{k}} r_{j, k} u_{j, k}=: r_{n}, \\
\left\|x^{(n)}-\left(x^{+}+P_{V}^{A}\left(x^{(0)}\right)\right)\right\|_{2}^{2}=\sum_{k=1}^{m} \prod_{s=0}^{n-1}\left|1-\lambda_{s} \mu_{k}\right|^{2} \sum_{j=1}^{p_{k}}\left|r_{j, k}\right|^{2} .
\end{gathered}
$$

(b) Assume that the relaxation coefficients are chosen such that $0 \leq \lambda_{n} \rho^{2} \leq 2$ for $n \geq 0$.

Then the iteration $x^{(n)}$ generated by the Landweber scheme (3) converges to a solution of the normal equations (6) for any $b \in \mathbf{K}^{M}$ and any initial guess $x^{(0)} \in \mathbf{K}^{N}$ if

$$
\sum_{n=0}^{\infty} a_{1, n}=+\infty .
$$

Under the conditions, the limit of the iteration $x^{(n)}$ is equal to $x^{+}+P^{A}\left(x^{(0)}\right)$.

(c) For the semi-positive definite singular matrix $A^{*} A$, the iteration $x^{(n)}$ generated by the Landweber scheme (3) with $\lambda_{n}=\frac{2}{\mu_{\max }+\mu_{\min }}(n=1,2, \cdots)$ is divergent and does not converge to $x^{+}+P^{A}\left(x^{(0)}\right)$.

Proof. (a) and (b) were already proved in $[7,21]$.

Since the matrix $A^{*} A$ is semi-positive definite and singular, its' smallest eigenvalue $\mu_{\min }=0$ and the relaxation coefficient $\lambda_{n}=\frac{2}{\mu_{\max }}(n=1,2, \cdots)$. By $(20)$,

$$
\begin{aligned}
x^{(n)}-x^{+}-P^{A}\left(x^{(0)}\right)-(-1)^{(n+1)} \sum_{j=1}^{p_{1}} r_{j, 1} u_{j, 1}= \\
-\sum_{k=2}^{m} \prod_{s=0}^{n-1}\left(1-\frac{2}{\mu_{\max }} \mu_{k}\right) \sum_{j=1}^{p_{k}} r_{j, k} u_{j, k} \longrightarrow 0 \text {, as } n \longrightarrow \infty .
\end{aligned}
$$

Thus, the iteration $x^{(n)}$ generated by the Landweber scheme (3) with $\lambda_{n}=\frac{2}{\mu_{\max }+\mu_{\min }}(n=1,2, \cdots)$ is divergent and does not converge to the solution $x^{+}+P^{A}\left(x^{(0)}\right)$.

\section{Relaxation Strategy}

The optimal and accelerating convergence schemes are proposed in this section. From (3), (11) and (14), if we let $z^{(n)}=x^{(n)}-x^{+}-P^{A}\left(x^{(0)}\right), H_{n}=\left(I-\lambda_{n} A^{*} A\right)$, then

$$
z^{(n+1)}=H_{n} z^{(n)}
$$

One sufficient condition for the iteration convergence is $\rho\left(H_{n}\right)=\left\|H_{n}\right\|_{2}<1$ [22]. This condition is fulfilled with the relaxation coefficients $\lambda_{n}$ being chosen by the following lemma.

Lemma 3.1. The successive approximation iteration given by (24) is non-expansive if

$$
0 \leq \lambda_{n} \leq \frac{2}{\mu_{1}} .
$$

Proof. By (9), the eigenvalues of $H_{n}$ are $1-\lambda_{n} \mu_{1}, \cdots, 1-\lambda_{n} \mu_{m}, 1, \cdots, 1$. Moreover, since $H_{n}$ is a Hermitian matrix, we get

$$
\rho\left(H_{n}\right)=\max _{1 \leq j \leq m}\left\{\left|1-\lambda_{n} \mu_{j}\right|, 1\right\} .
$$


If $\lambda_{n}<0$ or $\lambda_{n}>\frac{2}{\mu_{1}}$, we have $\left|1-\lambda_{n} \mu_{1}\right|>1$ and so $\rho\left(H_{n}\right)>1$. If $0 \leq \lambda_{n} \leq \frac{2}{\mu_{1}}$, then $\left|1-\lambda_{n} \mu_{1}\right| \leq 1$. Since $\mu_{1}>\mu_{2}>\cdots>\mu_{m}$, we get

$$
0 \leq \lambda_{n} \leq \frac{2}{\mu_{1}}<\frac{2}{\mu_{j}}, \quad\left|1-\lambda_{n} \mu_{j}\right| \leq 1,
$$

for $j=2,3, \cdots, m$. Therefore, for $0 \leq \lambda_{n} \leq \frac{2}{\mu_{1}}$,

$$
\rho\left(H_{n}\right)=\max _{1 \leq j \leq m}\left\{\left|1-\lambda_{n} \mu_{j}\right|, 1\right\} \leq 1 .
$$

For the selection of relaxation scheme, we have the following two theorems. By (13) of the Theorem 2.1, we define the error vector as $z^{(n)}:=x^{(n)}-x^{+}-P^{A}\left(x^{(0)}\right)$ of n-th step iteration for (3) and

$$
G_{n-1}=U \operatorname{Diag}\left(\left(1-\lambda_{n-1} \mu_{1}\right) I_{p_{1}}, \cdots,\left(1-\lambda_{n-1} \mu_{m}\right) I_{p_{m}}, O_{q}\right) U^{*}
$$

Then

$$
z^{(n)}=G_{n-1} z^{(n-1)}, n=1,2, \cdots,
$$

where $z^{(0)}=U r$. We define the matrix $G_{n-1}$ as the iterative matrix .

Theorem 3.2. For a initial guess $x^{(0)} \in \mathbf{R}^{N}$, the optimal relaxation strategy means that the spectral radium of the iterative matrix $G_{n-1}$ achieves its minimum. Thus, the optimal relaxation strategy for the Landweber iteration (3) is

$$
\lambda_{\text {opt }}=\frac{2}{\mu_{m}+\mu_{1}}, \quad \text { for } n=1,2, \cdots .
$$

where $\mu_{m}$ and $\mu_{1}$ are the smallest positive and biggest eigenvalues of the matrix $A^{*} A$ respectively. Proof. Let $\rho(G(\lambda))=\max _{1 \leq j \leq m}\left\{\left|1-\lambda \mu_{j}\right|\right\}$, then

$$
\rho\left(G\left(\lambda_{n-1}\right)\right)=\left\|G_{n-1}\right\| .
$$

Since $\mu_{1}>\mu_{2}>\cdots>\mu_{m}>0$, we have

$$
1-\lambda \mu_{1}<1-\lambda \mu_{2}<\cdots<1-\lambda \mu_{m} .
$$

Therefore we get

$$
\rho(G(\lambda))=\max \left\{\left|1-\lambda \mu_{1}\right|,\left|1-\lambda \mu_{m}\right|\right\} .
$$

Since

$$
\left|1-\lambda \mu_{j}\right|= \begin{cases}1-\lambda \mu_{j}, & \text { for } 0<\lambda \leq \frac{1}{\mu_{j}} \\ \lambda \mu_{j}-1, & \text { for } \lambda>\frac{1}{\mu_{j}}\end{cases}
$$

for $j=1, m$. By (34), we obtain

$$
\rho(G(\lambda))= \begin{cases}\max \left\{1-\lambda \mu_{1}, 1-\lambda \mu_{m}\right\}=1-\lambda \mu_{m}, & \text { for } 0<\lambda \leq \frac{1}{\mu_{1}} \\ \max \left\{\lambda \mu_{1}-1,1-\lambda \mu_{m}\right\}, & \text { for } \frac{1}{\mu_{1}}<\lambda<\frac{1}{\mu_{m}} \\ \max \left\{\lambda \mu_{1}-1, \lambda \mu_{m}-1\right\}=\lambda \mu_{1}-1, & \text { for } \lambda \geq \frac{1}{\mu_{m}}\end{cases}
$$

and

$$
\rho(G(\lambda))=\max \left\{\lambda \mu_{1}-1,1-\lambda \mu_{m}\right\}, \quad \lambda>0 .
$$

The optimal $\lambda_{\text {opt }}$ is reached at the cross point of the line $\lambda \mu_{1}-1$ and the line $1-\lambda \mu_{m}$. Let

$$
\lambda \mu_{1}-1=1-\lambda \mu_{m}
$$


we obtain the optimal relaxation coefficient is

$$
\lambda_{\text {opt }}=\frac{2}{\mu_{1}+\mu_{m}}=\arg _{\lambda} \min \rho(G(\lambda)), n=1,2, \cdots .
$$

and the corresponding spectral radius is

$$
\rho\left(G\left(\lambda_{\text {opt }}\right)\right)=\min _{\lambda} \rho(G(\lambda))=\frac{\mu_{1}-\mu_{m}}{\mu_{1}+\mu_{m}}<1 .
$$

Lemma 3.3. For any $\lambda^{(1)} \in\left(0, \frac{1}{\mu_{1}}\right)$, its symmetric point about $\lambda=\frac{1}{\mu_{1}}$ is $\lambda^{(2)}=\frac{2}{\mu_{1}}-\lambda^{(1)}$, where $\lambda^{(2)} \in\left(\frac{1}{\mu_{1}}, \frac{2}{\mu_{1}}\right)$ and

$$
\rho\left(G\left(\lambda^{(1)}\right)\right)>\rho\left(G\left(\lambda^{(2)}\right)\right)
$$

Proof. By (36) and (37), we obtain

$$
\rho(G(\lambda))=\max \left\{\lambda \mu_{1}-1,1-\lambda \mu_{m}\right\}= \begin{cases}1-\lambda \mu_{m}, & \text { for } 0<\lambda<\frac{2}{\mu_{1}+\mu_{m}} \\ \lambda \mu_{1}-1, & \text { for } \lambda \geq \frac{2}{\mu_{1}+\mu_{m}}\end{cases}
$$

and so we have

$$
\rho\left(G\left(\lambda^{(1)}\right)\right)=1-\lambda^{(1)} \mu_{m}, \text { for } 0<\lambda^{(1)}<\frac{1}{\mu_{1}} .
$$

For $\lambda^{(2)}=\frac{2}{\mu_{1}}-\lambda^{(1)} \in\left(\frac{1}{\mu_{1}}, \frac{2}{\mu_{1}}\right)$, if $\lambda^{(2)}<\frac{2}{\mu_{1}+\mu_{m}}$, then

$$
\rho\left(G\left(\lambda^{(2)}\right)\right)=1-\lambda^{(2)} \mu_{m}<1-\lambda^{(1)} \mu_{m}=\rho\left(G\left(\lambda^{(1)}\right)\right) .
$$

If $\lambda^{(2)}>\frac{2}{\mu_{1}+\mu_{m}}$, then

$$
\rho\left(G\left(\lambda^{(2)}\right)\right)=\lambda^{(2)} \mu_{1}-1=1-\lambda^{(1)} \mu_{1}<1-\lambda^{(1)} \mu_{m}=\rho\left(G\left(\lambda^{(1)}\right)\right) .
$$

Therefore,

$$
\rho\left(G\left(\lambda^{(1)}\right)\right)>\rho\left(G\left(\lambda^{(2)}\right)\right), \text { for } \lambda^{(1)} \in\left(0, \frac{1}{\mu_{1}}\right), \lambda^{(2)}=\frac{2}{\mu_{1}}-\lambda^{(1)} .
$$

For a large matrix $A$, the computation of the smallest positive eigenvalue $\mu_{m}$ is time consuming. Because $\mu_{1}=\|A\|_{2}^{2}$, we can get it by calculating $\|A\|_{2}^{2}$. For the sparse matrix $A$, the method of computing the upper bound of the biggest eigenvalue of $A^{*} A$ is proposed in [25]. By (31) and Lemma 3.3, the smaller $\left\|G_{n}\right\|$ is, the faster $x^{(n)}$ converges to $x^{+}+P^{A}\left(x^{(0)}\right)$. In addition, according to Lemma 3.3, if a relaxation coefficient $\lambda^{(1)} \in\left(0, \frac{1}{\mu_{1}}\right)$ is chosen, let $\lambda^{(2)}=\frac{2}{\mu_{1}}-\lambda^{(1)}$, then (41) holds. Hence, the relaxation coefficients also satisfy the convergence condition of Theorem 2.2 (b) and the inequality $\frac{1}{\mu_{1}} \leq \lambda_{n}<\frac{2}{\mu_{1}}$. In this case, one strategy of relaxation coefficient is that $\lambda_{1}=\frac{1}{\mu_{1}}$ and $\lambda_{n}$ tends to $\frac{2}{\mu_{1}}$ monotonically as $n$ tends to $\infty$. By this strategy, the convergence speed of $x^{(n)}$ converging to $x^{+}+P^{A}\left(x^{(0)}\right)$ is expected to be faster than the strategy $0<\lambda_{n}<\frac{1}{\mu_{1}}$ in Lemma 3.3.

\section{Relaxation strategy for the Richardson iteration}

For the Richardson's iteration method, the optimal relaxation coefficient is known to be $\frac{2}{\mu_{\min }+\mu_{\max }}$ for the symmetric positive definite matrix $A$, where $\mu_{\min }$ and $\mu_{\max }$ are the smallest and biggest 
eigenvalues of $A$ [24]. When the underlying matrix is nonnegative definite matrix, using the results of Section III, we can obtain a generalization for the optimal relaxation coefficient for the Richardson's iteration method.

Consider the linear system

$$
A x=b,
$$

where $A$ is a real symmetric nonnegative matrix and (47) is consistent, $b \in R(A)$. If we replace the nonnegative definite matrix $A^{*} A$ in the Landweber scheme (3) with a symmetric nonnegative definite matrix $A$, the general Richardson's iteration is

$$
x^{(n+1)}=x^{(n)}+\lambda_{n}\left(b-A x^{(n)}\right) .
$$

If we let $\bar{b}=A^{*} b, \bar{A}=A^{*} A$, then the Landweber scheme (3) can be transformed to the Richardson's iteration. In the following of this section, we obtain the generalization for the optimal relaxation coefficient for the Richardson's iteration method by replacing $A^{*} A$ by $A$ in Section 2 . If the linear systems (47) is consistent, then $b \in R(A)$ and

$$
b=\sum_{k=1}^{m} \sum_{j=1}^{p_{k}} c_{j, k}^{*} u_{j, k}
$$

The corresponding minimization 2-norm solution of (47) is

$$
x_{b}^{+}=\sum_{k=1}^{m} \frac{1}{\mu_{k}} \sum_{j=1}^{p_{k}} c_{j, k}^{*} u_{j, k},
$$

i.e.

$$
A x_{b}^{+}=b .
$$

The initial guess $x^{(0)}$ can be decomposed as

$$
x^{(0)}=\sum_{k=1}^{m} \sum_{j=1}^{p_{k}} c_{j, k}^{(0)} u_{j, k}+x_{0}^{(0)}=x_{1}^{(0)}+x_{0}^{(0)},
$$

with $c_{j, k}^{(0)} \in \mathbf{K}$, for $1 \leq k \leq m$ and $1 \leq j \leq p_{k}$, and where $x_{1}^{(0)} \in R(A), x_{0}^{(0)} \in \mathcal{N}(A)$. The representation of the iteration $x^{(n)}$ generated by the Richardson's iteration is characterized in the following corollary.

Corollary 4.1. For $n=1, \cdots$, the $n$-th iteration $x^{(n)}$ generated by the Richardson's iteration (48) satisfies

$$
\begin{gathered}
x^{(n)}-x_{b}^{+}-x_{0}^{(0)}=\prod_{s=0}^{n-1}\left(I-\lambda_{s} A\right)\left(x_{1}^{(0)}-x_{b}^{+}\right) \\
x^{(n)}-x_{b}^{+}-x_{0}^{(0)}=U \operatorname{Diag}\left(\prod_{s=0}^{n-1}\left(1-\lambda_{s} \mu_{1}\right) I_{p_{1}}, \cdots, \prod_{s=0}^{n-1}\left(1-\lambda_{s} \mu_{m}\right) I_{p_{m}}, O_{q}\right) r,
\end{gathered}
$$

where $r=\left(r_{1,1}, \cdots, r_{1, p_{1}}, \cdots, r_{m, 1}, \cdots, r_{m, p_{m}}, 0, \cdots, 0\right)^{T} \in \mathbf{K}^{N}$ with $r_{j, k}=c_{j, k}^{*}-c_{j, k}^{(0)}$ for $1 \leq j \leq$ $p_{k}, 1 \leq k \leq m$ and $p=p_{1}+\cdots+p_{m}$. 
By the same proof as the Landweber iteration, the optimal relaxation strategy can be obtained for the Richardson's iteration. Let $z^{(n)}:=x^{(n)}-x^{+}-x_{0}^{(0)}$, and

$$
\begin{gathered}
G_{n-1}=U \operatorname{Diag}\left(\left(1-\lambda_{n-1} \mu_{1}\right) I_{p_{1}}, \cdots,\left(1-\lambda_{n-1} \mu_{m}\right) I_{p_{m}}, O_{q}\right) U^{*}, \\
z^{(n-1)}=\operatorname{Diag}\left(\prod_{s=0}^{n-2}\left(1-\lambda_{s} \mu_{1}\right) I_{p_{1}}, \cdots, \prod_{s=0}^{n-2}\left(1-\lambda_{s} \mu_{m}\right) I_{p_{m}}, O_{q}\right) r,
\end{gathered}
$$

then the equation (54) can be described as the following form

$$
z^{(n)}=G_{n-1} z^{(n-1)}
$$

where $z^{(0)}=U r$. We define the matrix $G_{n-1}$ as the iterative matrix. The vector $z^{(n)}$ is called as the error vector. Consequently, by Theorem 3.2, we can easily deduce the following corollary.

Corollary 4.2. Suppose matrix $A$ is a symmetric nonnegative definite, then for the initial guess $x^{(0)} \in \mathbf{R}^{N}$, the optimal relaxation strategy means that the spectral radium of iterative matrix $G_{n-1}$ achieves the minimum. Thus the optimal relaxation strategy about the general Richardson's iteration (48) is

$$
\lambda_{n}=\frac{2}{\mu_{m}+\mu_{1}}, \text { for } n=0,1,2, \cdots
$$

where $\mu_{m}$ and $\mu_{1}$ are the minimal positive and maximal eigenvalues of $A$.

When matrix $A$ is symmetric positive definite, Corollary 4.2 is the same result in [19]. Hence, Corollary 4.2 is a generalization of the optimal relaxation coefficient for the Richardson's iteration method in [19].

\section{Numerical Simulation}

In this section, firstly, we use the Cimmino's method to verify the effectiveness of the results of Theorem 3.2 and the accelerating convergence relaxation strategies proposed in Section 3. Secondly, we use the Cimmino's method and the Diagonal Relaxed Orthogonal Projection (DROP) method, to demonstrate the advantages of our relaxation strategies by comparing with the existing strategies. The Cimmino's simultaneous projection method [10] is as follows,

$$
x_{j}^{(n+1)}=x_{j}^{(n)}+\lambda_{n} \sum_{i=1}^{M} \frac{1}{M} \frac{A_{i, j}^{*}}{\left\|A^{i}\right\|^{2}}\left(b^{i}-A^{i} x^{(n)}\right),
$$

where $j=1,2, \cdots, N$. Let $V=I_{N \times N}$ and $W=\frac{1}{M} \operatorname{Diag}\left(1 /\left\|A^{1}\right\|^{2}, \cdots, 1 /\left\|A^{M}\right\|^{2}\right)$, then (59) can be rewritten as

$$
x^{(n+1)}=x^{(n)}+\lambda_{n} V^{-1} A^{*} W\left(b-A x^{(n)}\right),
$$

where $W$ denotes the weights [3]. Besides, if let $V=\operatorname{diag}\left(M / N_{j}\right)$ where $N_{j}(j=1,2, \cdots, N)$ is the number of nonzeros in the jth column of $A$, then we obtain the simultaneous version of the DROP method $[3,27,28]$.

As we mentioned in Section 1, if we let $y^{(n)}=V^{\frac{1}{2}} x^{(n)}, D=W^{\frac{1}{2}} A V^{-\frac{1}{2}}, \bar{b}=W^{\frac{1}{2}} b$, then the weighted Landweber scheme (60) can be transformed to the Landweber scheme without weights

$$
y^{(n+1)}=y^{(n)}+\lambda_{n} D^{*}\left(\bar{b}-D y^{(n)}\right) .
$$




\subsection{Verification}

We give two examples about the image reconstruction from projections [24]. Using the SheppLogan phantom with $64 \times 64$ pixels and $256 \times 256$ pixels and the projections of 180 different angles, the pixels $x_{j}(j=1,2, \cdots, N)$ are labeled in a $1 \mathrm{D}$ sequential order and we get $x=\left(x_{1}, x_{2}, \cdots, x_{N}\right)^{T}$, where $N=64^{2}$ or $256^{2}$ and the same method for the projections $b_{i}(i=1,2, \cdots, M)$ we obtain $b=\left(b_{1}, b_{2}, \cdots, b_{M}\right)^{T}$, where $M=95$ or 367 . According to the method proposed in [26], we compute the coefficient matrix $A$ with order $95 \times 64^{2}$ or $367 \times 256^{2}$ respectively. In this experiment, the Cimmino's method with five different choices of $\lambda_{n}(n=0,1, \cdots)$ will be used to solve the linear systems.

In order to verify the reconstructed results, we calculate three kinds of image distance measurements: $d, r$ and $e$ [24]. We use $t_{u, v}$ and $r_{u, v}$ to denotes the element of the row $u$ and the column $v$ respectively in the original image and reconstructed image. And the formulae of image distance are as follows

$$
\begin{gathered}
d=\left(\left(\sum_{u=1}^{\sqrt{N}} \sum_{v=1}^{\sqrt{N}}\left(t_{u, v}-r_{u, v}\right)^{2}\right) /\left(\sum_{u=1}^{\sqrt{N}} \sum_{v=1}^{\sqrt{N}}\left(t_{u, v}-\bar{t}\right)^{2}\right)\right)^{\frac{1}{2}}, \\
r=\sum_{u=1}^{\sqrt{N}} \sum_{v=1}^{\sqrt{N}}\left|t_{u, v}-r_{u, v}\right| / \sum_{u=1}^{\sqrt{N}} \sum_{v=1}^{\sqrt{N}}\left|t_{u, v}\right|, \\
e=\max _{1 \leq i \leq[\sqrt{N} / 2], 1 \leq j \leq[\sqrt{N} / 2]}\left|T_{i, j}-R_{i, j}\right|,
\end{gathered}
$$

where

$$
\begin{gathered}
\bar{t}=\left(\sum_{i=1}^{\sqrt{N}} \sum_{j=1}^{\sqrt{N}} t_{i, j}\right) / N, \\
T_{i, j}=\frac{1}{4}\left(t_{2 i, 2 i}+t_{2 i+1,2 j}+t_{2 i, 2 j+1}+t_{2 i+1,2 j+1}\right), \\
R_{i, j}=\frac{1}{4}\left(r_{2 i, 2 i}+r_{2 i+1,2 j}+r_{2 i, 2 j+1}+r_{2 i+1,2 j+1}\right) .
\end{gathered}
$$

The criteria $d$ describes the overall nearness between the original and reconstructed image, the criteria $r$ is the mean relative error of reconstruction, the criteria $e$ is the maximum difference between the original and reconstructed image. The smaller the value of $\mathrm{d}$, r, e, the better the reconstructed results.

So for the first example, we can get the biggest and smallest positive eigenvalues of $D^{*} D$ : $\mu_{1}=0.5898, \mu_{m}=0.0426$. Therefore, we take the following five relaxation schemes to perform the simulations by iterating 10 steps. The $1-4$ schemes are to test the result of Theorem 3.2 . Since the biggest eigenvalue $\mu_{1}$ can always be obtained by calculation, we choose the scheme $\lambda_{n-1}=\frac{2}{x+\mu_{1}}$, where $x$ take some different values. The fifth scheme $\lambda_{n}=\frac{1}{\mu_{1}}\left(2-\frac{1}{n+1}\right)$ is based on the accelerating convergence relaxation strategies in section III and satisfies $\frac{1}{\mu_{1}}<\lambda_{n}<\frac{2}{\mu_{1}}$. For the second example, because we can not compute the smallest positive eigenvalue of $\left(D^{*} D\right)_{256^{2} \times 256^{2}}$, so we use the fifth scheme to implement simulations.

1) Scheme $1 \lambda_{n}=\frac{2}{0.50+\mu_{1}}$ for $n=0,1,2, \cdots, 10$. 
2) Scheme $2 \lambda_{n}=\frac{2}{0.35+\mu_{1}}$ for $n=0,1,2, \cdots, 10$.

3) Scheme $3 \lambda_{n}=\frac{2}{0.25+\mu_{1}}$ for $n=0,1,2, \cdots, 10$.

4) Scheme $4 \lambda_{n}=\frac{2}{\mu_{m}+\mu_{1}}$ for $n=0,1,2, \cdots, 10$.

5) Scheme $5 \lambda_{n}=\frac{1}{\mu_{1}}\left(2-\frac{1}{n+1}\right)$ for $n=0,1,2, \cdots, 10$.

Applying these five schemes to the Cimmino's simultaneous projection method (60), we obtain the reconstructions of the Shepp-Logan phantom with $N=64 \times 64$ pixels. The reconstructed results are as follows in Table1.

Table 1: Image distance comparison

\begin{tabular}{|c|c|c|c|c|}
\hline$\lambda_{n}$ & $\mathrm{~d}$ & $\mathrm{r}$ & $\mathrm{e}$ & time (s) \\
\hline Scheme 1 & 0.1748 & 0.1072 & 0.1145 & 93.9032 \\
\hline Scheme 2 & 0.1732 & 0.0998 & 0.1130 & 90.7124 \\
\hline Scheme 3 & 0.0974 & 0.0973 & 0.0858 & 87.5913 \\
\hline Scheme 4 & 0.0902 & 0.0900 & 0.0531 & 63.5615 \\
\hline Scheme 5 & 0.0974 & 0.0934 & 0.0601 & 69.3372 \\
\hline
\end{tabular}
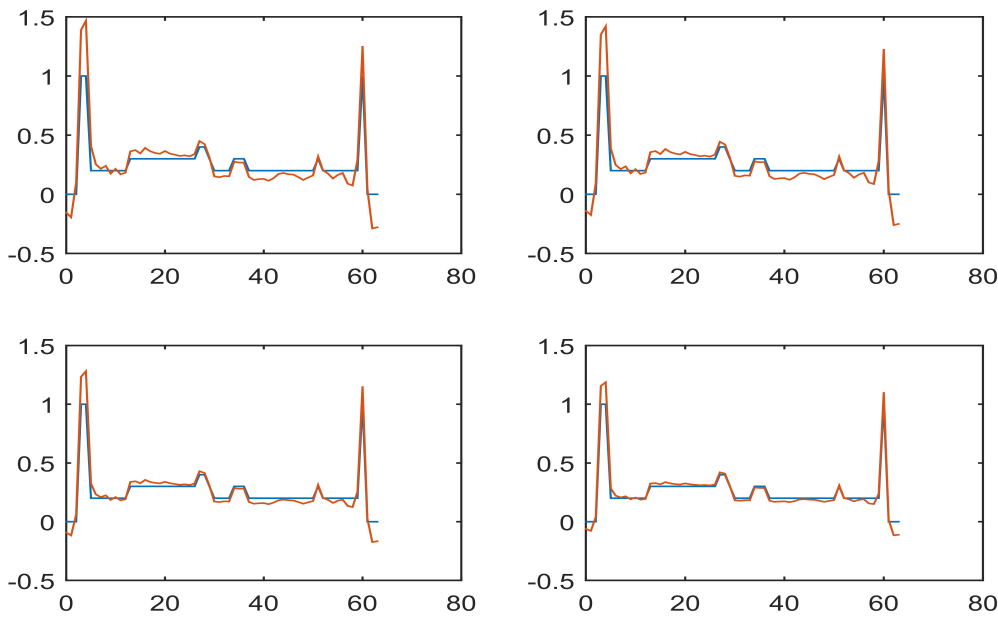

Figure 1: Comparison of the 32th column in the reconstruction and original image. The red line is reconstruction. Top row: using $\lambda_{n}=2 /\left(0.50+\mu_{1}\right)$ (left), using $\lambda_{n}=2 /\left(0.35+\mu_{1}\right)$ (right). Bottom row: using $\lambda_{n}=2 /\left(0.25+\mu_{1}\right)$ (left), using $\lambda_{n}=2 /\left(\mu_{m}+\mu_{1}\right)$ (right).

From the Table 1 and the Figure 1, we can see that with the relaxation coefficient close to $\frac{2}{\mu_{1}+\mu_{m}}$, the quality of the reconstruction is getting better and better, the reconstruction time gradually shorten. So the optimal scheme is $\lambda_{n}=\frac{2}{\mu_{1}+\mu_{m}}$, as given in the Theorem 3.2.

For the pixels of $256 \times 256$, we can only obtain the biggest eigenvalue. In this case, the importance of the fifth scheme is appeared. Applying the following five schemes to the Cimmino's simultaneous projection method (59), we also obtain the reconstruction of the Shepp-Logan phantom image with $N=256 \times 256$ pixels which is shown in Figure 2. The image distance comparison is in Table 2. Figure 3 illustrates the comparison of the 128th column in the reconstruction and original image. As we see, the reconstruction results verify the correctness of the Lemma 3.3.

1) Scheme $1 \lambda_{n}=\frac{2}{0.5+\mu_{1}}$ for $n=0,1,2, \cdots, 10$. 
2) Scheme $2 \lambda_{n}=\frac{2}{0.3+\mu_{1}}$ for $n=0,1,2, \cdots, 10$.

3) Scheme $3 \lambda_{n}=\frac{2}{0.15+\mu_{1}}$ for $n=0,1,2, \cdots, 10$.

4) Scheme $4 \lambda_{n}=\frac{2}{0.05+\mu_{1}}$ for $n=0,1,2, \cdots, 10$.

5) Scheme $5 \lambda_{n}=\frac{1}{\mu_{1}}\left(2-\frac{1}{n+1}\right)$ for $n=0,1,2, \cdots, 10$.

Table 2: Image distance comparison

\begin{tabular}{|c|c|c|c|c|}
\hline$\lambda_{n}$ & $\mathrm{~d}$ & $\mathrm{r}$ & $\mathrm{e}$ & time $(\mathrm{s})$ \\
\hline Scheme1 & 0.1901 & 0.2013 & 0.3011 & 133.1715 \\
\hline Scheme 2 & 0.1734 & 0.1811 & 0.3103 & 119.3091 \\
\hline Scheme3 & 0.1257 & 0.1655 & 0.2981 & 110.1573 \\
\hline Scheme4 & 0.0903 & 0.1419 & 0.2805 & 99.6512 \\
\hline Scheme5 & 0.0924 & 0.1508 & 0.2736 & 100.7103 \\
\hline
\end{tabular}
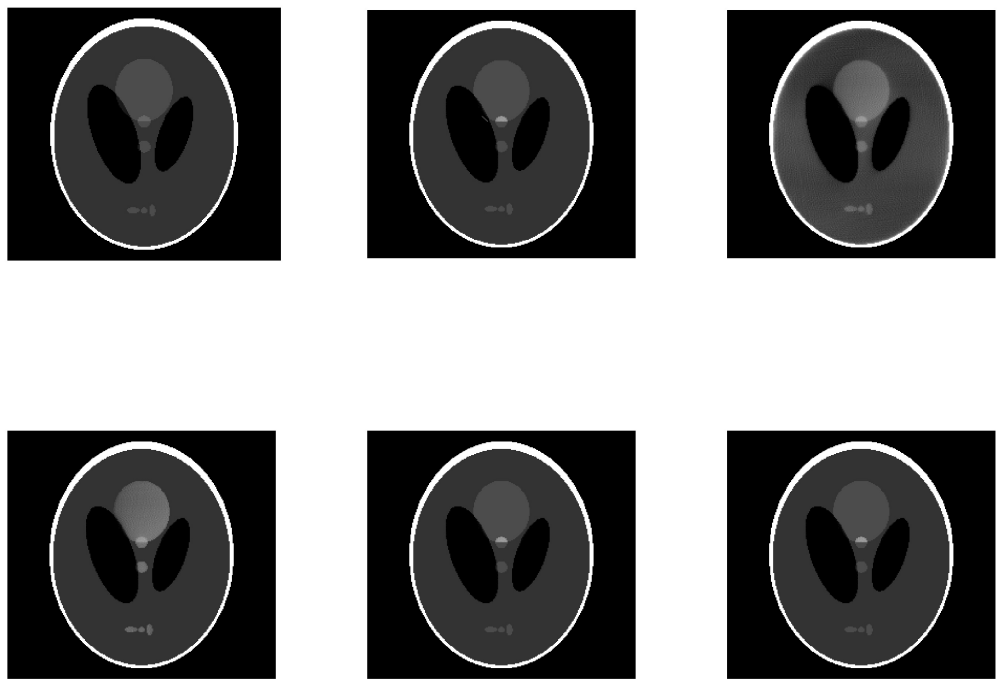

Figure 2: Reconstructed images of Shepp-Logan phantom by Cimmino's Simultaneous Projection method. Top row: Original image(left), Cimmino's reconstruction using $\lambda_{n}=2 /\left(0.5+\mu_{1}\right)$ (middle), using $\lambda_{n}=2 /\left(0.3+\mu_{1}\right)$ (right). Bottom row: using $\lambda_{n}=2 /\left(0.15+\mu_{1}\right)$ (left), using $\lambda_{n}=2 /\left(0.05+\mu_{1}\right)$ (middle), using $\lambda_{n}=(2-1 /(n+1)) / \mu_{1}$ (right).

\subsection{Comparison}

We compare our new strategies with the strategies proposed in [27,28], using an example taken from image reconstruction from projections with noise and noise-free. Here the Shepp-Logan phantom is discretized into $64 \times 64$ pixels, and we use 16 projections with 95 rays per projection. The matrix $A$ is computed using the method proposed in [26], which has the dimensions $1520 \times 4096$. The image pixels $x_{j}\left(j=1,2, \cdots, 64^{2}\right)$ are labeled in a $1 \mathrm{D}$ sequential order and then $x=\left(x_{1}, x_{2}, \cdots, x_{64^{2}}\right)^{T}$. The same method for all projections $b_{i}(i=1,2, \cdots, 1520)$, we get $b=\left(b_{1}, b_{2}, \cdots, b_{1520}\right)^{T}$. We add independent Gaussian noise $\delta b$ of mean 0 and various standard deviations to generate three different noise levels $\eta=\|\delta b\| /\|b\|=0.01,0.05$, and 0.08 , then the projection vector is $b+\delta b$. The exact solution $\bar{x}$ denotes the original image. Cimmino and DROP 

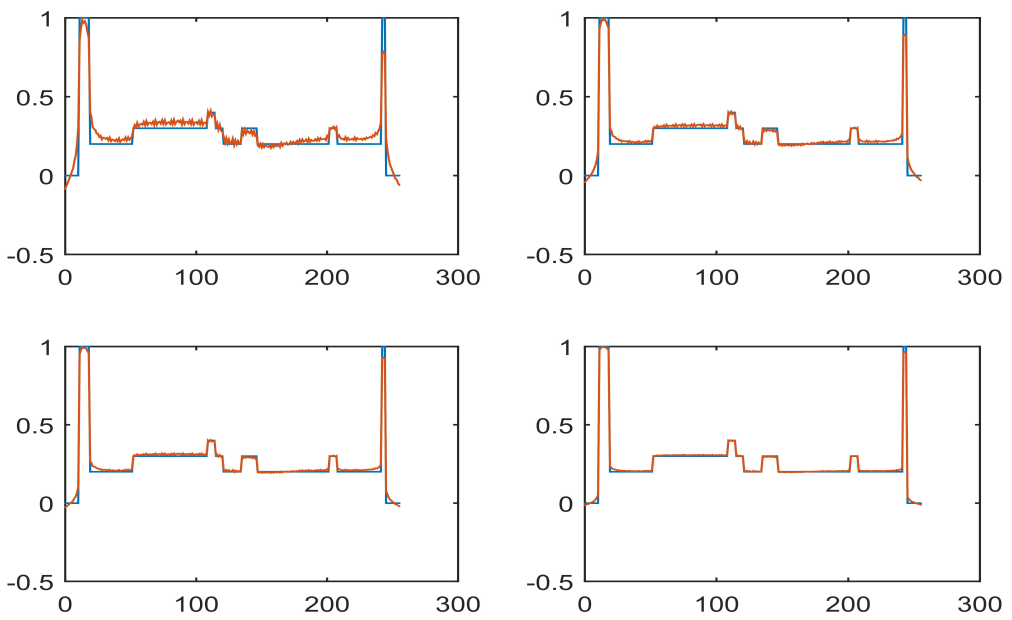

Figure 3: Comparison of the 128th column in the reconstruction and original image. The red line is reconstruction. Top row: using $\lambda_{n}=2 /\left(0.3+\mu_{1}\right)$ (left), using $\lambda_{n}=2 /\left(0.15+\mu_{1}\right)$ (right). Bottom row: using $\lambda_{n}=2 /\left(0.05+\mu_{1}\right)$ (left), using $\lambda_{n}=(2-1 /(n+1)) / \mu_{1}$ (right).
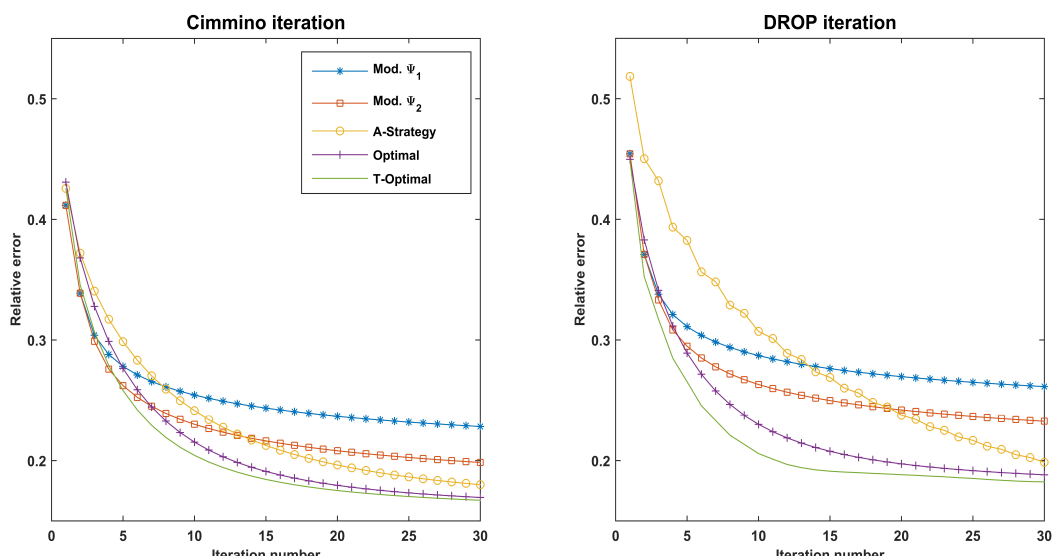

Figure 4: Relative error histories for the underdetermined system, for five different choices of $\lambda_{n}$.

methods are used to simulate. We take five different strategies, Modfied $-\Psi_{1}$, Modfied $-\Psi_{2}$, A-Strategy $\lambda_{n}=\frac{1}{\mu_{1}}\left(2-\frac{1}{n+1}\right)$ proposed in Section 3, Optimal $\left(\lambda_{n}=\frac{2}{\mu_{1}+\mu_{m}}\right)$ and T-Optimal. Here the T-Optimal strategy is based on picking $\lambda_{n}$ such that the error $\left\|\bar{x}-x^{(n)}\right\|_{2}$ is minimized in each iteration. The forms of the Modified $\Psi_{1}$ and $\Psi_{2}$ are as follows,

$$
\begin{array}{ll}
\text { Modified } \Psi_{1} \text {-rule }: & \lambda_{n}= \begin{cases}\sqrt{(2)} \rho^{-2}, & \text { for } n=0,1, \\
2 \tau^{(1)} \rho^{-2}\left(1-\zeta_{n}\right), & \text { for } n \geq 2,\end{cases} \\
\text { Modified } \Psi_{2}-\text { rule }: & \lambda_{n}= \begin{cases}\sqrt{(} 2) \rho^{-2}, & \text { for } n=0,1, \\
2 \tau^{(2)} \rho^{-2} \frac{1-\zeta_{n}}{\left(1-\zeta_{n}^{n}\right)^{2}}, & \text { for } n \geq 2,\end{cases}
\end{array}
$$

where $\tau^{(1)}=2$ and $\tau^{(2)}=1.5$ which are chosen heuristically to accelerate the convergence, $\rho=$ $\rho\left(A^{*} W A\right)$, with $W$ equal to the identity we get the Landweber iteration, and then $\rho=\mu_{1}, \zeta_{n}$ is the unique root in $(0,1)$ of the polynomial $[27,28]$

$$
g_{n-1}(y)=(2 n-1) y^{n-1}-\left(y^{n-2}+\cdots+y+1\right) .
$$


Figure 4 and Figure 5 show the relative errors in the reconstructions, defined as

$$
\text { Relative error }=\frac{\left\|\bar{x}-x^{(n)}\right\|_{2}}{\|\bar{x}\|_{2}},
$$

as functions of the iteration index $n[27,28]$.

As shown in the Figure 4, for the noise-free, the strategy T-Optimal is the best, but the exact solution must be known. The Optimal strategy based on minimizing the spectral radius of the iterative matrix and A-Strategy Scheme 5 are better than the strategies Mod. $\Psi_{1}$ and Mod. $\Psi_{2}$. For the projections with noise, from the Figure 5, we observe that for the Cimmino's method the strategies Mod. $\Psi_{1}$ and $\operatorname{Mod} . \Psi_{2}$ are better than our new strategies for not too small noise levels, but for the DROP method A-Strategy is better than Mod. $\Psi_{1}$ and $\operatorname{Mod} . \Psi_{2}$ with the increase of the number of the iterations. The proposed relaxation strategies are feasible and effective based on the theoretical deduction and experimental results.
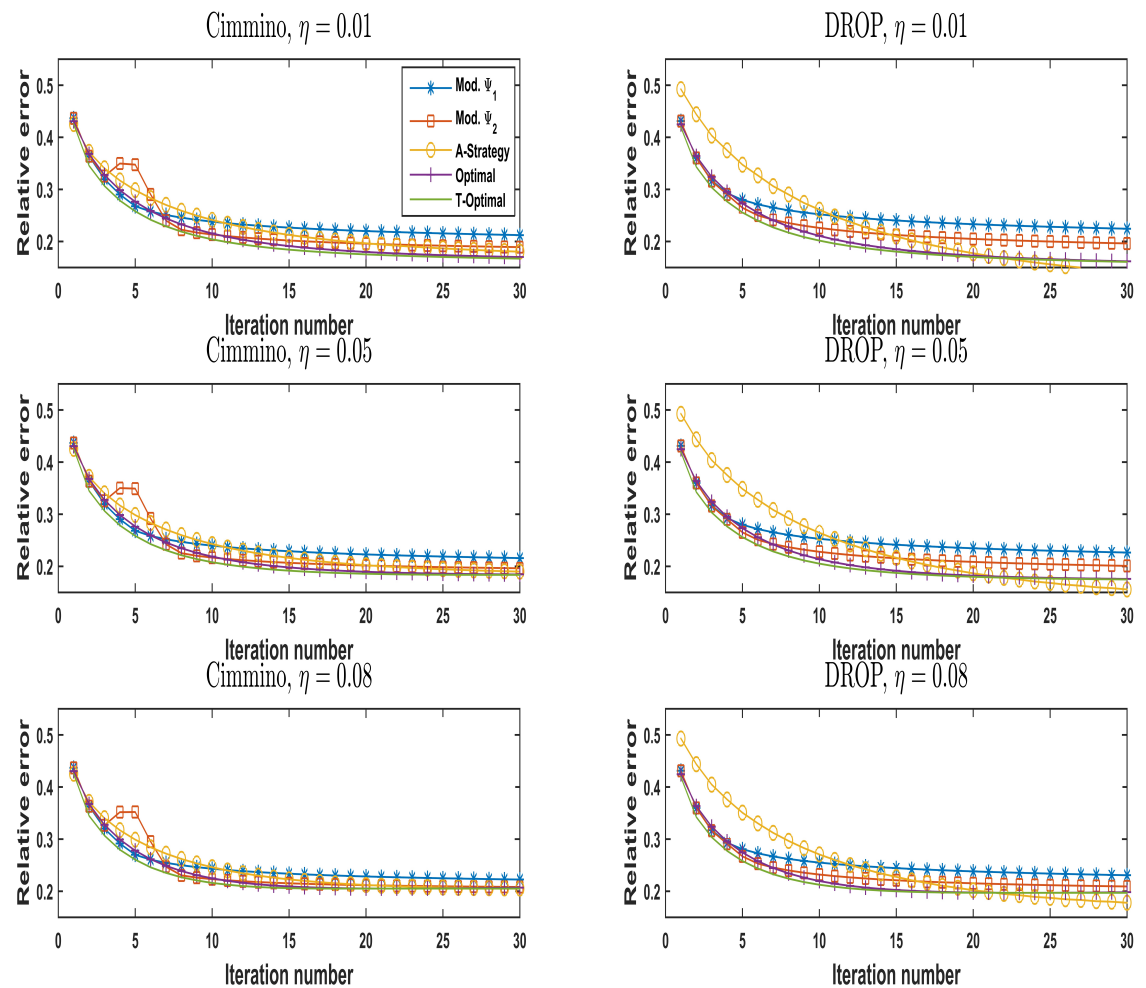

Figure 5: Relative error histories for the underdetermined system, for five different choices of $\lambda_{n}$ and three noise levels $\eta$.

\section{Conclusion}

Based on the derived iterative representation formula and the convergence results of the Landweber iteration, we have proposed the optimal relaxation coefficient $\lambda_{n}=2 /\left(\mu_{1}+\mu_{m}\right)(n=1,2, \cdots)$ under the spectral radius minimization of the new derived iterative matrix, where $\mu_{1}$ and $\mu_{m}$ are the largest and smallest positive eigenvalues of $A^{*} A$. If only $\mu_{1}$ is known, we have proposed the 
iterative relaxation strategies of accelerating convergence, $\frac{1}{\mu_{1}} \leq \lambda_{n}=\frac{1}{\mu_{1}}\left(2-\frac{1}{n+1}\right)<\frac{2}{\mu_{1}}$ and $\lambda_{n}$ satisfying the convergence condition for the Landweber method. By this strategy, the speed of $x^{(n)}$ converging to $x^{+}+P^{A}\left(x^{(0)}\right)$ is faster than the speed by the corresponding strategy $0<\lambda_{n}<\frac{1}{\mu_{1}}$.

\section{Acknowledgments}

M. Jiang is partially supported by the National Basic Research Program of China (973 Program) (2015CB351803), National Science Foundation of China (61421062, 61520106004), and SinoGerman Center (GZ 1025). G. Han and G. Qu is partially supported by the National Natural Science Foundations of China (61271012, 61071144).

[1] M. Bertero and P. Boccacci, Inverse Problems in Imaging. Bristol and Philadelphia: Institute of Physical Publishing, 1998.

[2] Y. Censor and T. Elfving, Block-iterative algorithms with diagonally scaled oblique projections for the linear feasibility problem, SIAM Journal on Matrix Analysis and Applications. 24 (2002) $40-58$.

[3] M. Jiang and G. Wang, Convergence studies on iterative algorithms for image reconstruction, IEEE Transactions on Medical Imaging. 22 (5) (2003) 569 - 579.

[4] M. Jiang, Iterative algorithms for image reconstruction, Medical Imaging Systems: Technology and Applications. C. Leondes, Ed., vol. I. Singapore: World Scientific. (2005) 351 - 382.

[5] G. Qu, and M. Jiang, Landweber scheme for compact operator equation in Hilbert, Communications in Numerical Methods in engineering. 25(6) (2009) 771 - 786.

[6] Y. Censor, T. Elfving, G. Herman, and T. Nikazad, On diagonally-relaxed orthogonal projection methods, SIAM Journal on Scientific Computing. 30 (2008) 473 - 504.

[7] G. Qu, C. Wang and M. Jiang, Necessary and Sufficient Convergence Conditions for Algebraic Image Reconstruction Algorithms, IEEE Transactions on Image Processing. 18 (2009) 435 440 .

[8] T. Elfving T. and T. Nikazad, Properties of a class of block-iterative methods, Inverse Problems. 25 (2009) 115011.

[9] A. H. Andersen and A. C. Kak, Simultaneous algebraic reconstruction technique (SART): a superior implementation of the ART algorithm, Ultrasonic Imaging. 6 (1984) 81 - 94 .

[10] G. Cimmino, Calcolo approssimato per le soluzioni dei sistemi diequazioni lineari, La Ricerca Scientifica XVI, series II, Anno IX. (1938) 326 - 333. 
[11] Y. Censor, D. Gordon and R. Gordon, Component averaging: an efficient iterative parallel algorithm for large and sparse unstructured problems, Parallel Computing. 27 (2001) 777 808.

[12] A. Dax, The convergence of linear stationary iterative processes for solving singular unstructured systems of linear equations, SIAM Review. 32 (4) (1990) 611 - 635.

[13] X. Shi, Y. Wei, and W. Zhang, Convergence of General Nonstationary Iterative Methods for Solving Singular Linear Equations, SIAM Journal on Matrix Analysis and Applications. 32 (2011) $72-89$.

[14] J. Wang and Y. Zheng, On the convergence of generalized simultaneous iterative reconstruction algorithms, IEEE Transactions on Image Processing. 16 (1) (2007) 1 - 6.

[15] A. Kirsch, An Introduction to the Mathematical Theory of Inverse Problems, ser. Applied Mathematical Sciences. Berlin - Heidelberg: Springer-Verlag, no. 120, 1996.

[16] G. Qu, C. Wang and R. Kang, Reconstruction of Band-limited Signals From Uniform Random Samples, Advances in Mathematics. 41 (2) (2012) 253 - 255.

[17] M. Eiermann, W. Niethammer and R. S. Varga, A study of semi-iterative methods for nonsymmetric systems of linear equations, Numerische Mathematik. 47 (4) (1985) 505 - 533.

[18] R. S. Varga, Matrix iterative analysis, expanded ed., ser. Springer Series in Computational Mathematics. Berlin: Springer-Verlag, 2000, vol. 27.

[19] Y. Saad and H. A. van der Vost, Iterative solution of linear systems in the 20th century, Journal of Computational and Applied Mathematics. 123 (2000) 1 - 33.

[20] G. M. Fichtenholz, Differential and integral calculus, II.2 (Chinese). (translated from the Russian). Beijing: People's Education Press, 1979.

[21] G. Qu, M. Jiang, Y. Yang, Convergence results of Landweber iterations for linear systems, Acta. Mathematicae Applicatae Sinica, English Series. 30 (1) (2014) 111-118.

[22] H. Wozniakowski, Round-off error analysis of iterations for large linear systems, Numerische Mathematik. 30 (1978) 301-314.

[23] Y. Saad, Iterative methods for sparse linear systems, PWS publishing, New York,1996.

[24] G. Herman, Image reconstruction from projections, Academic Press, INC,1980.

[25] Byrne C L. Applied iterative methods.[J]. A K Peters Ltd Wellesley Ma, 2008.

[26] R. L. Siddon, Fast calculation of the exact radiological path of a three-demensional CT array, Medical Physics. 12 (1985) 252-255. 
[27] J. Gregor, T. Benson, Computational Analysis and Improvement of SIRT, IEEE Trans. Med. Imaging. 27 (2008) 918-924.

[28] W. Qiu, J.R. Tong, C.N. Mitchell, T. Marchant, P. Spencer, C.J. Moore, M. Soleimani, New iterative cone beam CT reconstruction software: Parameter optimisation and convergence study, Computer methods and programs in biomedicine. 100 (2010) 166-174.

[29] P.C. Hansen, M.Saxild-Hansen, AIR Tools: A MATLAB package of algebraic iterative reconstruction methods, Journal of Computational and Applied Mathematics. 236 (2011) 21672178.

[30] T. Elfving, T. Nikazad and P.C. Hansen, Semi-convergence and relaxation parameters for a class of SIRT algorithms, Electronic Transactions on Numerical Analysis. 37 (2012) 321-336.

[31] T. Elfving, P. C. Hansen and T. Nikazad, Semiconvergence and relaxation parameters for projected SIRT algorithm, SIAM Journal on Scientific Computing. 34 (2012) A2000-A2017.

[32] L.T. Dos Santos, A parallel subgradient projections method for the convex feasibility problem, J. Comput. Appl. Math. 18 (1987) 307-320.

[33] G. Appleby and D. C. Smolarski, A linear acceleration row action method for projecting onto subspaces, Electron. Trans. Numer. Anal. 20 (2005) 253-275.

[34] A. Dax, Line search acceleration of iterative methods, Linear Algebra Appl. 130 (1990) 43-63.

[35] J. Gregor and J. A. Fessler, Comparison of SIRT and SQS for regularized weighted least squares image reconstruction, IEEE Transactions on Computational Imaging, 1 (2015) 44-55.

[36] C. Xu, D. Tao and C. Xu, Multi-view learning with incomplete views, IEEE Trans. Imaging Processing. 24 (2015) 5812-5825. 